\title{
In vivo, ex vivo and in vitro dasatinib activity in chronic lymphocytic leukemia
}

\author{
KRZYSZTOF GIANNOPOULOS $^{1 *}$, AGNIESZKA KARCZMARCZYK ${ }^{1 *}$, MARTA KARP $^{1 *}$, \\ AGNIESZKA BOJARSKA-JUNAK ${ }^{2}$, KAMILA KOSIOR $^{3}$, MALGORZATA KOWAL $^{3}$, \\ WALDEMAR TOMCZAK ${ }^{3}$, MAREK HUS $^{3}$, MARCIN MACHNICKI $^{4}$ and TOMASZ STOKŁOSA ${ }^{4}$ \\ Departments of ${ }^{1}$ Experimental Hematooncology, ${ }^{2}$ Clinical Immunology and \\ ${ }^{3}$ Hematooncology and Bone Marrow Transplantation, Medical University of Lublin, Lublin 20-093; \\ ${ }^{4}$ Department of Immunology, Center for Biostructure Research, Medical University of Warsaw, Warsaw 02-097, Poland
}

Received February 11, 2020; Accepted December 30, 2020

DOI: 10.3892/ol.2021.12546

\begin{abstract}
Dasatinib inhibits the breakpoint cluster region-Abelson murine leukemia $1(B C R-A B L 1)$ gene along with other kinases known to be overexpressed and abnormally active in patients with chronic lymphocytic leukemia (CLL). The current study used primary leukemic cells obtained from 53 patients with CLL that were treated with dasatinib. A 2,3-bis-(2-methoxy-4-nitro-5-sulfophenyl) -2H-tetrazolium-5-carboxanilide (XTT) assay and Annexin $\mathrm{V}$ staining was performed to assess the cytotoxic effects of dasatinib treatment. The XTT assay revealed that the median cytotoxicity of dasatinib was $8.30 \%$ (range, $0.00-77.89 \%$ ). Due to high dispersion of dasatinib activity, patients were divided into sensitive ( $\mathrm{n}=27 ; 50.94 \%$; median cytotoxicity, 22.81\%) and resistant groups ( $\mathrm{n}=26 ; 49.06 \%$; median cytotoxicity, $0.00 \%)$. A median cytotoxicity of $8.30 \%$ was selected as a cut off value. Using Annexin V staining and flow cytometry on exemplary sensitive and resistant CLL samples, it was revealed that 17.71 and $1.84 \%$ of cells were apoptotic, respectively. The current study presented a case of a patient with concomitant occurrence of CLL and chronic myeloid leukemia (CML) with a major molecular response after dasatinib treatment. A simultaneous reduction of circulating CLL cells indicated in vivo anti-CLL activity induced by dasatinib. After an in vitro culture of the patient's mononuclear cells with subsequent dasatinib treatment, a higher percentage of CLL cells undergoing apoptosis was obsevered when compared with untreated samples (38.19 vs. $21.99 \%$, respectively). Similarly,
\end{abstract}

Correspondence to: Professor Krzysztof Giannopoulos, Department of Experimental Hematooncology, Medical University of Lublin, Chodźki 1, Lublin 20-093, Poland

E-mail: krzysztof.giannopoulos@gmail.com

*Contributed equally

Key words: chronic lymphocytic leukemia, chronic myeloid leukemia, dasatinib, kinase inhibitor, apoptosis the percentage of CLL apoptotic cells $\left(\Delta \Psi \mathrm{m}^{\text {low }}\right)$ measured by chloromethyl-X-rosamine was higher after incubation with dasatinib $(7.28 \%)$ than in the negative control $(2.86 \%)$. In conclusion, dasatinib induced antileukemic effects against CML and CLL cells. The results of the current study indicated that dasatinib may induce apoptosis ex vivo, in vitro and in vivo in CLL.

\section{Introduction}

Chronic lymphocytic leukemia (CLL) is a heterogeneous disease with a number of markers used for patient risk stratification. The most commonly utilized in clinical practice are: i) deletion of chromosome arms17p and 11q, ii) TP53 and immunoglobulin heavy chain variable region $(I G H V)$ genes mutations as well as iii) protein expression of the zeta chain associated protein-70 (ZAP-70) and CD38 (1-3).

Since CLL is a malignancy of B lymphocytes, the role of signaling through $\mathrm{B}$-cell receptor (BCR) and microenvironment stimulation seems to be crucial in the pathogenesis of the disease $(4,5)$. The inhibition of BCR signaling by targeting Bruton's tyrosine kinase (BTK) or phosphatidylinositol 3 kinase (PI3K) led to durable remissions and prolonged progression-free and overall survival even in CLL patients with negative prognostic features. Despite those advances in CLL therapy, there is still a group of relapsed and/or refractory patients, therefore, the need to investigate novel treatment options still exists (6). CLL cell survival is driven by overexpression and abnormal activity of several non-receptor tyrosine kinases, members of Src family kinase (SFK)-LYN, SYK, and c-ABL tyrosine kinase $(4,7,8)$. It was proven that inhibition of c-ABL or LYN/SYK kinases with specific tyrosine kinase inhibitors (TKI) induces apoptosis of CLL cells. Thus targeting particular kinases might be a promising option for CLL therapy (4,9-12).

Dasatinib is a second generation inhibitor of breakpoint cluster region-Abelson murine leukemia 1 (BCR-ABL1) kinase approved in 2006 by the Food and Drug Administration in the treatment of chronic myeloid leukemia (CML) and chromosome Philadelphia-positive acute lymphoblastic leukemia (ALL) patients (13). Dasatinib is a multiple kinases 
inhibitor with significant activity against tyrosine kinases that are known to be important in the pathogenesis of several hematological malignancies and solid tumors. Low concentrations of dasatinib have been shown to inhibit not only BCR-ABL1, but also c-KIT, platelet-derived growth factor receptor (PDGFR), BTK, TEC, LYN, and other SFK. In CLL, increased SFK and c-ABL kinases activity was reported, giving thereby a rationale to evaluate dasatinib antileukemic activity in CLL $(6,14,15)$. In vitro studies showed that dasatinib also inhibits anti-apoptotic proteins that are overexpressed in CLL, namely Bcl-xL, Bf1/A1 and Mcl-1 (16). Furthermore, this inhibitor contributes to a reduction of cytoskeletal activity by its interaction in the LYN/HS1 pathways $(17,18)$. Moreover, we found earier, that dasatinib might target similar genes as thalidomide-antiangiogenic agent with proven antileukemic activity in CLL (19).

It was previously reported that dasatinib at a concentration of $5 \mu \mathrm{M}$ was able to induce apoptosis in ZAP-70 positive CLL patients with unmutated $I G H V$ genes (4). Moreover, Amrein et al (15) showed that CLL lymphocytes with del17 were highly sensitive to dasatinib when compared with CLL lymphocytes without del17 (median $\mathrm{IC}_{50}$ values of 0.1 and $34 \mu \mathrm{M}$, respectively). Meanwhile, other groups demonstrated that dasatinib in nanomolar concentrations induced 50\% inhibition of SFK kinases causing significant apoptosis of CLL cells disregard of prognostic markers (8,9,20-22).

Dasatinib activity in CLL remains unclear. Moreover, there is a lack of consistent data regarding which group of patients could benefit most from therapy with this TKI. Therefore, we aimed to evaluate the effect of dasatinib in vitro in a cohort of 53 CLL patients. Moreover, as a proof of concept, we present the detailed ex vivo and in vivo analyses of dasatinib activity in a patient with concomitant CML and CLL who is being successfully treated with dasatinib until date.

\section{Materials and methods}

Patients. Peripheral blood and bone marrow samples were obtained from 53 patients diagnosed with CLL and a patient diagnosed with CLL and CML at the Department of Hematooncology and Bone Marrow Transplantation, Medical University of Lublin, Poland. Table I summarizes the clinical characteristics of CLL patients. The study was approved by the Ethics Committee of the Medical University of Lublin (nos. KE-0254/269/2019 and KE-0254/305/2019).

Cell isolation. Peripheral blood mononuclear cells (PBMCs) and bone marrow mononuclear cells (BMMCs) were isolated by Ficoll (Biochrom AG) density gradient centrifugation. The viability of cells was evaluated by Trypan blue staining (Sigma-Aldrich; Merck KGaA) and quantified in a Neubauer chamber (Zeiss GmbH).

RNA extraction and reverse transcription. Total RNA was extracted using QIAamp RNA BloodMini Kit (Qiagen) according to the manufacturer's instructions. The concentration and purity of isolated RNA were determined using spectrophotometer BioSpec-nano Micro-volume UV-Vis (Shimadzu). From each sample, $2 \mu \mathrm{g}$ of total RNA was reverse transcribed to cDNA using SuperScript III First-Strand Synthesis System for RT-PCR (Thermo Fisher Scientific, Inc.) and Veriti Dx 96-Well Thermal Cycler (Thermo Fisher Scientific, Inc.).

Quantitative reverse transcriptase polymerase chain reaction (qRT-PCR) for BCR-ABL1. Quantitative analysis of BCR-ABL1 gene expression was performed according to European Leukemia Net and EUTOS standards for monitoring a TKI therapy in CML (23). Two separate reaction mixtures providing amplification of $B C R-A B L 1$ fusion gene and Abelson $(A B L)$ control gene were prepared. The following primers and probes were used: 5'-TCCGCTGACCATCAATAAGGA-3', forward primer BCR-ABL1; 5'-CACTCAGACCCTGAGGCTCAA-3', reverse primer $B C R-A B L 1 ; 5$ 'FAM-CCCTTCAGCGGCCAGTAGCATC TGA-3'TAMRA, probe $B C R$ - $A B L 1$; 5'-TGGAGATAACACTCT AAGCATAACTAAAGG-3', forward primer $A B L ;$ 5'-GATGTA GTTCTTGGGACCCA-3', reverse primer $A B L ;$ 5'FAM-CCATTT TTGGTTTGGGCTTCACACCATT-3' TAMRA, probe $A B L$. The qRT-PCR reaction was performed on the 7500 Fast Dx Real-Time PCR instrument (ThermoFisher Scientific, Inc.), using $5 \mu \mathrm{l}$ cDNA, gene-specific primers, molecular probe and TaqMan ${ }^{\circledR}$ Universal PCR Master Mix (ThermoFisher Scientific, Inc.) in $25 \mu \mathrm{l}$ end volume. Thermocycling program was set for 50 cycles of $2 \mathrm{~min}$ at $50^{\circ} \mathrm{C}, 10 \mathrm{~min}$ at $95^{\circ} \mathrm{C}, 15 \mathrm{sec}$ at $95^{\circ} \mathrm{C}, 60 \mathrm{sec}$ at $60^{\circ} \mathrm{C}$. Quantitative analysis of $B C R-A B L 1$ and $A B L$ gene expression was performed using a calibration curve prepared by serial dilutions of BCR-ABL1 Mbcr Standards and $A B L$ Control Gene 3 Standards for a known $B C R-A B L 1$ and $A B L$ copy number. $B C R-A B L 1$ copy numberwas normalized to $A B L . B C R-A B L 1 / A B L$ ratio expressed as a percentage (\%) was calculated. The result was multiplied by laboratory-specific conversion factor (CF) and expressed using International Scale (IS).

Analyses of SF3B1, NOTCH1, MYD88 L265P and IGHV mutations. Genomic DNA was isolated from PBMCs using QIAamp DNA BloodMini Kit (Qiagen) according to the manufacturer's recommendation. Detection of $\mathrm{SF} 3 \mathrm{~B} 1, \mathrm{NOTCH} 1$ and MYD88 L265P mutation, and $I G H V$ mutation status were assessed as described earlier in detail (24). A cutoff of $98 \%$ germline homology was used to assess $I G H V$ mutation status. The sequences with a germline homology of $98 \%$ or higher were considered unmutated and those with a homology $<98 \%$ were considered mutated.

Immunophenotypic analysis. The immunophenotypic analysis was performed by flow cytometry. In our study, the standard diagnostic flow cytometric analysis included monoclonal antibodies (MoAbs) anti-CD5-FITC/CD19-PE, anti-CD19-FITC and anti-CD23-PE (BD Biosciences). A standard, whole-blood assay with erythrocyte cell lysis was used for preparing the peripheral blood specimens. The samples were analyzed by flow cytometry directly after preparation. For data acquisition and analysis, a FACSCalibur instrument (Becton-Dickinson and Company) with CellQuest software (Becton Dickinson and Company) was used. For each analysis, 10,000 events were acquired and analyzed. The percentage of positive cells was measured from a cut-off set using isotype-matched nonspecific control antibody. Evaluation

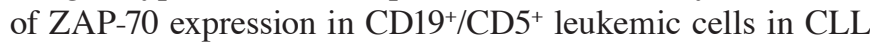
samples was performed as previously described (25). Flow cytometric analysis of CD38 on leukemic cells was performed on PB samples using monoclonal antibodies: anti-CD19 FITC, 
Table I. Clinical characteristics of patients.

\begin{tabular}{|c|c|}
\hline Characteristic & $\mathrm{N}(\%)$ \\
\hline \multicolumn{2}{|l|}{ Age (years) } \\
\hline Median & 65 \\
\hline Range & $47-84$ \\
\hline \multicolumn{2}{|l|}{$\operatorname{Sex}$} \\
\hline Female & $33(62.26)$ \\
\hline Male & $20(37.74)$ \\
\hline \multicolumn{2}{|l|}{ Rai stage } \\
\hline 0 & $22(41.51)$ \\
\hline I & $13(24.53)$ \\
\hline II & $8(15.09)$ \\
\hline III & $3(5.66)$ \\
\hline IV & $3(5.66)$ \\
\hline Not available & $4(7.55)$ \\
\hline \multicolumn{2}{|l|}{ WBC $\left(\times 10^{9} / 1\right)$} \\
\hline Median & 28.8 \\
\hline Range & $3.70-144.00$ \\
\hline \multicolumn{2}{|l|}{ LDH (IU/l) } \\
\hline Median & 384 \\
\hline Range & $258-961$ \\
\hline \multicolumn{2}{|l|}{ B2M (mg/l) } \\
\hline Median & 2.76 \\
\hline Range & $1.61-9.49$ \\
\hline \multicolumn{2}{|l|}{ ZAP-70 (cut-off 20\%) } \\
\hline Positive & $30(56.60)$ \\
\hline Negative & $12(22.64)$ \\
\hline Not available & $11(20.76)$ \\
\hline \multicolumn{2}{|l|}{ CD38 (cut-off 30\%) } \\
\hline Positive & $11(20.75)$ \\
\hline Negative & $35(66.04)$ \\
\hline Not available & $7(13.21)$ \\
\hline \multicolumn{2}{|c|}{$I G H V$ mutational status } \\
\hline Mutated & $15(28.30)$ \\
\hline Unmutated & $15(28.30)$ \\
\hline Not available & $23(43.40)$ \\
\hline \multicolumn{2}{|l|}{ Cytogenetics } \\
\hline del11q & $2(3.77)$ \\
\hline del13q & $8(15.10)$ \\
\hline del17p & $2(3.77)$ \\
\hline Normal karyotype & $12(22.64)$ \\
\hline Not available & $29(54.72)$ \\
\hline \multicolumn{2}{|c|}{ NOTCH1 mutational status } \\
\hline Mutated & $2(3.77)$ \\
\hline Unmutated & $20(37.74)$ \\
\hline Not available & $31(58.49)$ \\
\hline \multicolumn{2}{|c|}{ SF3B1 mutational status } \\
\hline Mutated & $0(0.00)$ \\
\hline Unmutated & $28(52.83)$ \\
\hline Not available & $25(47.17)$ \\
\hline \multicolumn{2}{|c|}{ MYD88 L265P mutational status } \\
\hline Mutated & $0(0.00)$ \\
\hline Unmutated & $24(45.28)$ \\
\hline Not available & $29(54.72)$ \\
\hline
\end{tabular}

WBC, white blood cells count; LDH, lactate dehydrogenase; ZAP-70, zeta-chainassociated protein kinase 70; CD, cluster of differentiation; IGHV, immunoglobulin heavy chain variable region; B2M, $\beta 2$-mikroglobulin; Del, deletion; NOTCH1, neurogenic locus notch homolog protein 1; MYD88, myeloid differentiation primary response gene 88; SF3B1, splicing factor 3B subunit 1 .
anti-CD38 PE and anti-CD5 PE-Cy5 (BD Pharmingen). The cut-off for positivity of leukemia cells for ZAP-70 expression was $\geq 20 \%$, while for CD38 was $\geq 30 \%$.

Routine laboratory results such as white blood cell count (WBC), lactate dehydrogenase level (LDH) and B2M ( $\beta 2$-mikroglobulin), and cytogenetic and FISH analyses of CLL patients were accessed from the hospital laboratory at the first admission.

In vitro cytotoxicity assay with XTT dye. The cytotoxic effect of dasatinib was measured using in vitro 2,3-bis-(2-m ethoxy-4-nitro-5-sulfophenyl)-2H-tetrazolium-5-carboxanilide (XTT)-based method (Sigma-Aldrich; Merck KGaA). PBMCs were suspended in X-VIVO w/o Phenol red and Genatamycin (Lonza) and added on a 96-well plate at a concentration of $5 \times 10^{5}$ cells $/ 100 \mu \mathrm{l} /$ well. Dasatinib at a final concentration of $180 \mathrm{nM}$, reflecting plasma drug concentrations observed in a clinical setting, was added to experimental wells. As a negative control, only live cells were used, positive control consisted of cells treated with $0.1 \%$ Triton X-100 (Sigma-Aldrich; Merck KGaA). Twenty-five microliters of XTT reagent was added to all samples. Plates were incubated for $24 \mathrm{~h}$ in a humidified atmosphere with $5 \% \mathrm{CO}_{2}$ at $37^{\circ} \mathrm{C}$. Optical densities (OD) were measured at $450 \mathrm{~nm}$ with a VICTOR3 1420 multilabel counter (PerkinElmer, Inc.), as a background wavelength at $690 \mathrm{~nm}$ was used. Each sample was performed in triplicates. Cytotoxic effect was calculated as below: cytotoxicity $=\left[1-\left(\mathrm{OD}_{\mathrm{s}}-\mathrm{OD}_{\mathrm{b}}\right) /\left(\mathrm{OD}_{\mathrm{c}}-\mathrm{OD}_{\mathrm{b}}\right)\right] \times 100 \%$, where: ODs is an OD of assayed sample, ODb is an OD of positive control and ODc is an OD of live cells (negative control).

Cell culture. The PBMCs of a patient diagnosed with CLL and CML were incubated at a concentration of $2 \times 10^{6}$ cells $/ \mathrm{ml}$ for $24 \mathrm{~h}$ with $180 \mathrm{nM}$ of dasatinib and without dasatinib in a control sample. Cells were incubated in a standard medium consisting of RPMI-1640 (Biochrom, Berlin, Germany) supplemented with $50 \mathrm{U} / \mathrm{ml}$ penicillin, $50 \mu \mathrm{g} / \mathrm{ml}$ streptomycin, $100 \mu \mathrm{g} / \mathrm{ml}$ neomycin and $10 \%$ heat-inactivated fetal bovine serum at $37^{\circ} \mathrm{C}$ in a humidified atmosphere of $5 \% \mathrm{CO}_{2}$. After cell culture, the percentage of apoptotic cells within $\mathrm{CD} 19^{+}$ cells was measured using flow cytometry.

Apoptosis analyses. The percentage of apoptotic cells was measured after $24 \mathrm{~h}$ incubation with $180 \mathrm{nM}$ of dasatinib and compared to non-treated samples. Two methods were used for apoptosis assessment:

i) Annexin V staining of CLL samples and CLL/CML patient. The analysis was performed using Annexin V-FITC Apoptosis Detection Kit (Sigma-Aldrich; Merck KGaA) according to the manufacturer's instructions. PBMCs were washed with PBS (Biochrom AG), suspended in a binding buffer provided and stained with $5 \mu 1$ of Annexin V-FITC. The PBMCs were incubated for $10 \mathrm{~min}$ in the darkness and immediately analyzed on a FACSCalibur (BD Biosciences).

ii) Mito Tracker Red CMXRos (Molecular Probes) technique used on CLL/CML patient. Chloromethyl-X-rosamine (CMXRos) is a cationic lipophilic fluorochrome that does not accumulate in depolarised mitochondria and thus can be used to detect disruptions in the mitochondrial membrane potential $(\Delta \Psi \mathrm{m})$. PBMCs were incubated with CMXRos for $30 \mathrm{~min}$ 

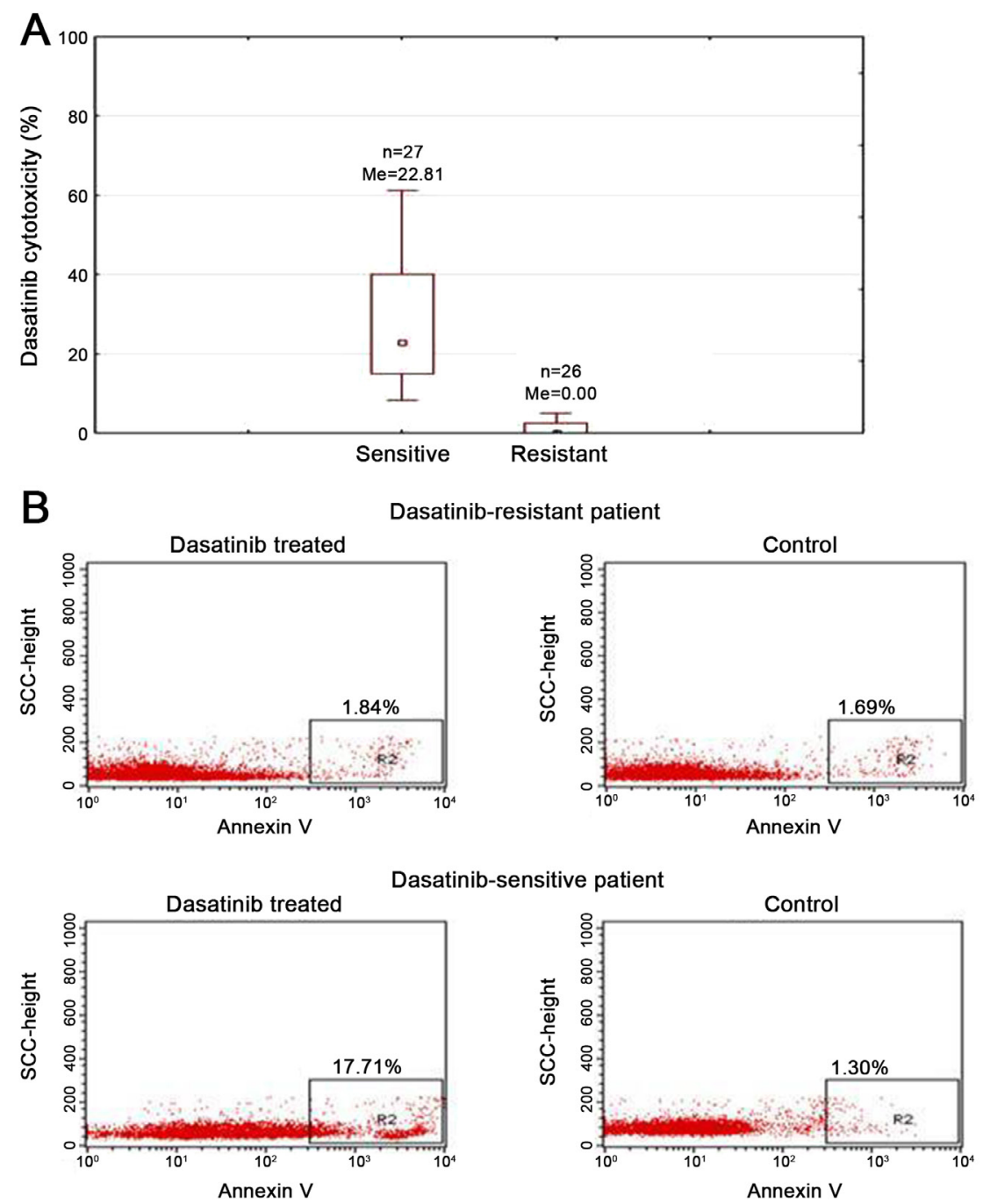

Figure 1. Response of patients with chronic lymphocytic leukemia after treatment with $180 \mathrm{nM}$ dasatinib for $24 \mathrm{~h}$. (A) Dasatinib cytotoxicity among sensitive and resistant patients evaluated using a 2,3-bis-(2-methoxy-4-nitro-5-sulfophenyl)-2H-tetrazolium-5-carboxanilide test. (B) Apoptosis analysis of dasatinib resistant and sensitive patients. In each graph, the R2 quadrant represents apoptotic cells. Me, median; SSC, side scatter.

at $37^{\circ} \mathrm{C}$ and analyzed on a FACSCalibur. Cells considered to be apoptotic displayed a decrease in mitochondrial membrane potential in CMXRos staining $\left(\Delta \Psi \mathrm{m}^{\text {low }}\right)$.

The collapse of $\Delta \Psi \mathrm{m}$ is a marker of early apoptosis, preceding other hallmarks of cell death, such as DNA fragmentation or phosphatidylserine externalisation (detected by Annexin V).

Statistical analysis. Statistical analysis was performed using STATISTICA 12 program (StatSoft Polska Sp. z o. o.). All results are presented as median values with range. The Mann-Whitney U test was used to evaluate the differences between subgroups of patients. Correlations of variables were calculated with the Spearman rank correlation coefficient.

\section{Results}

Dasatinib induces cytotoxic effect of CLL cells. To assess the cytotoxic effect of dasatinib on primary CLL cells, we performed XTT test. Freshly isolated PBMCs of $53 \mathrm{CLL}$ patients were incubated with $180 \mathrm{nM}$ of dasatinib for $24 \mathrm{~h}$. Observed median cytotoxicity of dasatinib was $8.3 \%$ and it varied between individual cases (range: $0-77.89 \%$ ). In 10 patients $(18.87 \%)$ obtained dasatinib cytotoxicity was exceeding $30 \%$. The cytotoxicity between $20-30 \%$ was observed in 7 patients $(13.21 \%)$. In 9 patients $(16.99 \%)$ dasatinib cytotoxicity ranged from 10 to $20 \%$. In 27 cases $(50.93 \%)$ the cytotoxicity was $<10 \%$. Due to high dispersion of dasatinib activity, we have divided analyzed group into sensitive $(n=27,50.94 \%$, median cytotoxicity: $22.81 \%)$ and resistant patients ( $n=26,49.06 \%$, median cytotoxicity: $0 \%)$ using median cytotoxicity of $8.3 \%$ as a cut off value (Fig. 1A).

Dasatinib cytotoxicity correlates with $\beta 2$-microglobulin levels. To identify patients who will benefit most from dasatinib therapy, we correlated the dasatinib activity with CLL prognostic factors. Statistical analysis revealed a positive correlation of dasatinib cytotoxicity with $\beta 2$-microglobulin serum levels $(\mathrm{R}=0.317 ; \mathrm{P}=0.041)$. We observed no correlations between dasatinib activity and white blood cell count, lactate dehydrogenase level or age of patients.

Dasatinib cytotoxicity in different prognostic subgroups. We observed a lack of differences in dasatinib cytotoxicity 
in different stages of CLL, according to Rai classification $(\mathrm{P}=0.823)$. Rai stage 0 patients displayed the median cytotoxicity of dasatinib of $14.87 \%$ (range: $0-59.18 \%, n=22$ ). In Rai stage I the median cytotoxicity of dasatinib was $7.46 \%$ (range: $0-36.90 \%, \mathrm{n}=13$ ). A similar result was observed in cases with Rai stage II-median: $7.36 \%(\mathrm{n}=8$; range: $0-77.89 \%)$. In groups with Rai stage III $(n=3)$ and IV $(n=3)$ the median dasatinib cytotoxicities were $10.92 \%$ (range: $0-46.22 \%, n=3$ ) and $10.59 \%$ (range: $0.51-49.31 \%, \mathrm{n}=3$ ), respectively. We also found no significant differences of dasatinib activity depending on the sex of patients: Among women dasatinib cytotoxicity was $14.73 \%$ (range: $0-61.14 \%, \mathrm{n}=33$ ), while in group of men $6.61 \%$ (range: $0-77.89 \% ; \mathrm{n}=20, \mathrm{P}=0.442$ ). The effect of dasatinib on CLL primary cells was independent of CD38 ( $\mathrm{P}=0.837)$ as well as ZAP-70 expression ( $\mathrm{P}=0.404)$. Among $\mathrm{CD} 38$ and ZAP-70 positive cases median TKI cytotoxicity was $10.59 \%$ (range: $0-77.89 \%, \mathrm{n}=11$ ) and $18.93 \%$ (range: $0-77.89 \%, \mathrm{n}=12$ ), in cohorts assigned as CD38 and cases ZAP-70 negative we observed median cytotoxicity of $14.27 \%$ (range: $0-61.14 \%$, $\mathrm{n}=35$ ) and $9.58 \%$ (range: $0-61.14 \%, \mathrm{n}=30$ ), respectively. We also observed no differences in dasatinib response depending on the mutation status of $I G H V$ genes $(\mathrm{P}=0.901)$. In the group with unmutated $I G H V$ genes median dasatinib cytotoxicity was $17.76 \%$ (range: $0-77.89 \%, \mathrm{n}=15$ ), likewise to patients with mutated $I G H V$ genes the median of $15.02 \%$ (range: $0-61.14 \%$, $\mathrm{n}=15$ ). Similarly, there were no statistical differences in the dasatinib effect in subgroups with different cytogenetic abnormalities $(\mathrm{P}=0.826)$. Mean cytotoxicity in cases with del11q was $4.29 \%$ (range: $4.13-4.46 \%, \mathrm{n}=2$ ) and in patients with del17p was $2.66 \%$ (range: $0-4.13 \%, \mathrm{n}=2$ ). In patients with del13q median cytotoxicity of dasatinib was $10.05 \%$ (range: $0-61.14 \%, \mathrm{n}=8$ ). Among patients with normal karyotype dasatinib cytotoxicity was $5.68 \%$ (range: $0-77.89 \%, \mathrm{n}=12$ ). Moreover, we analyzed dasatinb response in regard to the new prognostic factors of CLL, namely mutations in NOTCH1, SF3B1 and MYD88 genes. All tested samples did not have SF3B1 $(\mathrm{n}=28)$ nor MYD88 mutation $(\mathrm{n}=24)$. Of 22 examined cases 2 had mutation in NOTCH1 and 20 were unmutated with median cytotoxicity of 29.85 and $2.74 \%$, respectively, but the difference in dasatanib cytotoxicity between these subgroups was not of statistical significance $(\mathrm{P}=0.074)$.

Dasatinib induces apoptosis of CLL cells in vitro. To analyze induction of apoptosis after $24 \mathrm{~h}$ incubation with dasatinib Annexin V staining by flow cytometry on the representative sensitive or resistant to dasatinib cases (based on XTT results) was performed. We observed 17.71 and $1.84 \%$ of apoptotic cells, respectively for sensitive or resistant samples (Fig. 1B). In non-treated control cells the percentage of Annexin $\mathrm{V}$ positive cells was 1.30 and $1.69 \%$, respectively (Fig. 1B).

Case report. In January 2008, a 76-year-old man was diagnosed with CLL in Rai stage II. Bone marrow aspiration showed $70-90 \%$ infiltration by small mature-appearing lymphocytes carrying a clonal immunophenotype of $\mathrm{CD} 19^{+} / \mathrm{CD} 20^{+} / \mathrm{CD} 23^{+} / \mathrm{CD}^{+}$. A flow cytometry analysis of the patient's peripheral blood revealed a monoclonal B-cell population (92\%) positive for CD5, CD19, CD23. CD38 and ZAP-70 expressions were classified as negative (i.e. CD38 expression below cut-off value of $30 \%$ and ZAP-70 below 20\%). The
$I G H V$ gene mutation status was mutated. No chromosomal abnormalities were detected in the peripheral blood cells. At that time, disease was not active with no indications to start the treatment. In February 2009, the patient was referred to our institution with symptoms of active disease and was qualified to start therapy. He was treated with 6 cycles of fludarabine and cyclophosphamide (FC) chemotherapy. After completion of therapy, the patient had achieved a complete response.

In October 2013, leukocytosis with neutrocytosis was detected in peripheral blood. The white blood cell count (WBC) was $19.62 \times 10^{9} / 1$ (61\% of neutrophils, $6 \%$ of eosinophils, $1 \%$ of monocytes, $2 \%$ of band cells, $19 \%$ of lymphocytes, $9 \%$ of myeloblasts, $2 \%$ of promyelocytes), hemoglobin was $14.9 \mathrm{~g} / \mathrm{dl}$ and platelets were $163 \times 10^{9} / 1$. Bone marrow examination revealed hypercellularity, increased percentage of granulocytic lineage and the percentage of lymphocytes was $5 \%$. Karyotype was as follows: 46,XY, $\mathrm{t}(9 ; 22)(\mathrm{q} 34 ; \mathrm{q} 11.2)(19)$. The cytogenetic analysis showed a Philadelphia chromosome in $100 \%$ of cells. Fluorescence in situ hybridization (FISH) analysis was performed on the same cytogenetic sample. It showed a standard $B C R-A B L 1$ rearrangement in $96 \%$ of interphase nuclei. RT-PCR showed a $B C R-A B L 1$ b3a2 transcript. The level of transcript $[B C R-A B L 1 \times C F]$ was $80 \%$. Based on these findings, the diagnosis of the second malignancy-Ph+CML was made. In November 2013 imatinib at a dose of $400 \mathrm{mg}$ /day was started. After two weeks, imatinib was stopped due to muscle and osteoarticular system pains. Based on the literature suggesting the effectiveness of dasatinib in CLL, we proposed to replace imatinib with dasatinib in an attempt to control both diseases with monotherapy. Dasatinib was introduced at a dose of $100 \mathrm{mg} /$ day in December 2013. After 2 weeks, dasatinib treatment was suspended for 3 weeks due to pleural effusion. Steroids and diuretics have been used for pleural effusion management. After 1 month of dasatinib treatment, normalization of the peripheral blood hematological values was noticed. After seven months of therapy, the pleural effusion occurred again. Dasatinib dose reduction (from 100 to $50 \mathrm{mg}$ ) was required. In January 2015, after 12 months of dasatinib treatment, the patient exhibited major molecular response. This response has been maintained until the time of this report, with continued dasatinib therapy. Patient required no subsequent therapy for CLL. Fig. 2 displays changes in $B C R-A B L 1$ transcript, $\mathrm{WBC}$ and CLL cells $\left(\mathrm{CD} 23^{+} / \mathrm{CD} 19^{+} / \mathrm{CD}^{+}\right)$from January 2008 to November 2019 (last observation).

Antileukemic effect of dasatinib in CLL. In this study, we assessed whether dasatinib could induce apoptosis of leukemic cells ex vivo of the abovementioned CLL/CML patient. To assess apoptosis, we used both Annexin V and CMXRos stainings. In the present assay, the mononuclear cells did not exhibit of $B C R-A B L 1$ transcript.

We found that the percentage of apoptotic cells $\left(\mathrm{CD} 19^{+} /\right.$Annexin $\left.\mathrm{V}^{+}\right)$after in vitro incubation with dasatinib in a $24 \mathrm{~h}$ culture was higher than that in the culture without dasatinib (38.19 vs. $21.99 \%$, respectively). Dot plots, illustrating the

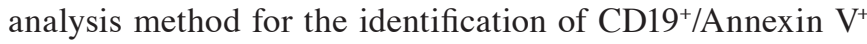
cells are shown in Fig. 3A. Similarly, the percentage of apoptotic cells $\left(\Delta \Psi \mathrm{m}^{\text {low }} / \mathrm{CD} 19^{+}\right)$measured by CMXRos was higher after incubation with dasatinib (7.28\%) than in negative control (2.86\%) (Fig. 3B). 
A
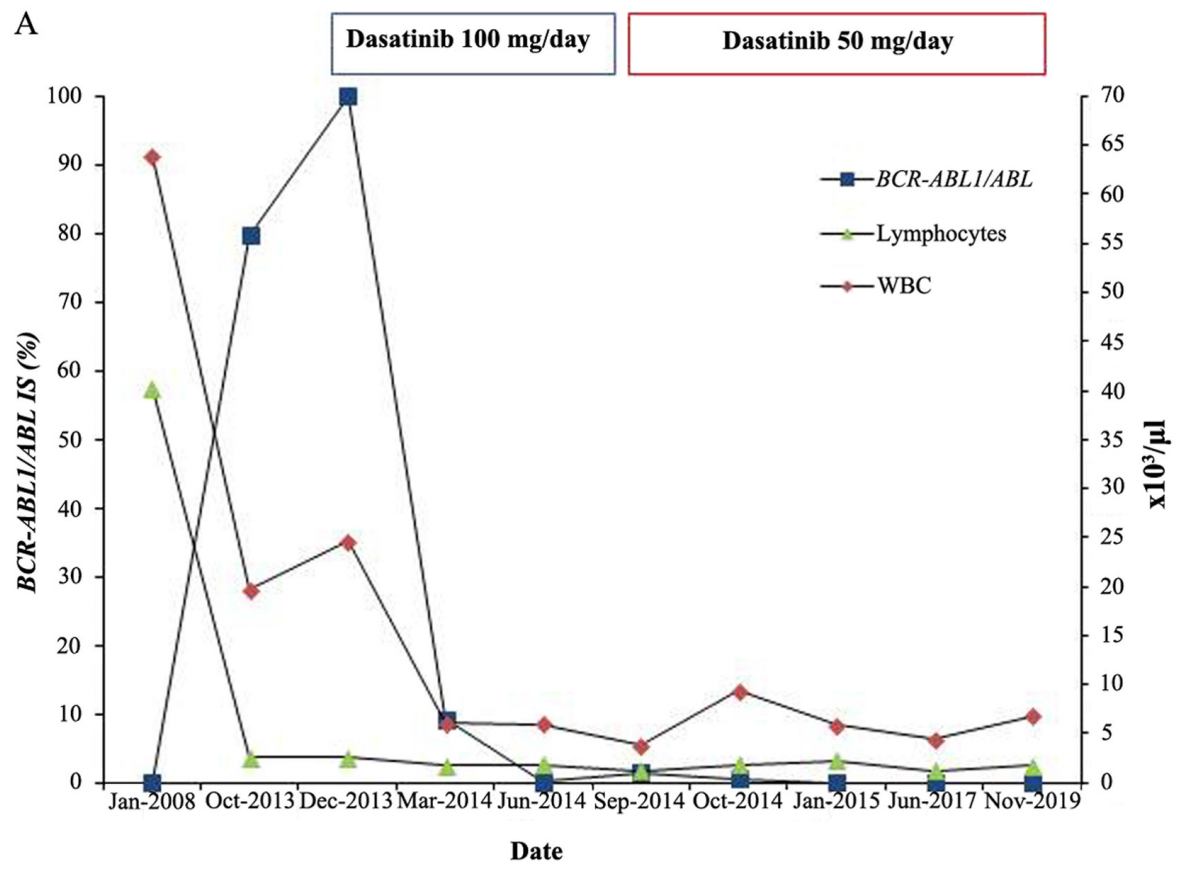

B

\begin{tabular}{|l|c|c|c|c|c|}
\hline & Jan-2008 & Oct-2013 & Oct-2014 & Jun-2017 & Nov-2019 \\
\hline Date & & & & & \\
\hline CD23+/CD19+/CD5+(x10/4) & 57.380 & 2.630 & 1.550 & 1.030 & 1.640 \\
\hline BCR-ABL1/ABL (\% IS) & & & & & \\
\hline
\end{tabular}

Figure 2. Laboratory test results performed during the observation of a patient with chronic lymphocytic leukemia/chronic myeloid leukemia. (A) White blood cell, absolute lymphocyte and the BCR-ABL1 transcript levels were determined using the International Scale during dasatinib treatment. (B) The absolute $\mathrm{CD} 23^{+} / \mathrm{CD} 19^{+} / \mathrm{CD}^{+}$count and the $B C R-A B L 1$ transcript level before and during dasatinib treatment was also calculated. WBC, white blood cell; BCR-ABL1, breakpoint cluster region-Abelson murine leukemia 1; IS, international scale.

\section{Discussion}

Over the past years, highly active novel therapies, including kinase inhibitors targeting BTK or PI3K, were implemented in the treatment of CLL patients. This approach is of high specificity, although inhibition of these kinases affects the whole signaling via BCR and, in consequence, multiple cellular processes (26).

The mechanism underlying CLL development remains unclear, but it is thought that some stimulus (unknown antigen: exogenous, autologous or antigen-independent cell-autonomous signaling) of BCR in combination with genetic, cytogenetic and epigenetic abnormalities leads to abnormal activation of multiple signaling pathways (27). In vitro activity of dasatinib on the apoptosis of CLL cells has been reported in patients who were ZAP-70 positive, with unmutated $I G V H$ genes, as well as with del17p or del11q $(9,28,29)$. Our in vitro results point to the preferential effectiveness of dasatinib in CLL cells, regardless of theses prognostic factors, though its cytotoxicity correlates with $\beta 2$-microglobulin serum levels. Moreover, Amrein et al (9) showed that addition of dasatinib at a concentration of $0.1 \mu \mathrm{mol} / 1$ sensitizes CLL cells to chlorambucil and fludarabine. While preclinical reports were encouraging, in phase 2 clinical trials dasatinib demonstrated moderate activity in relapsed CLL. Of 13 relapsed CLL patients, who received $50 \mathrm{mg}$ of dasatinib twice daily in phase 2 clinical study, 3 presented a decrease of lymphocyte count and regression of nodal disease (30). In another trial dasatinib was administered in a dose of $150 \mathrm{mg}$ once a day to patients refractory to fludarabine-based therapies, partial responses were observed in 3 out of 15 cases and 9 patients showed a nodal response. These findings confirmed the modest activity of dasatinib in monotherapy with acceptable toxicity (22). In a phase 2 study combining fludarabine $\left(40 \mathrm{mg} / \mathrm{m}^{2} /\right.$ day, days 1-3 every 28 day) and dasatinib (100 mg/day, days 1-28) in fludarabine-refractory CLL patients, the overall response rate was $18 \%$, but the clinical outcome of patients was significantly improved. Interestingly, temporary lymphocytosis in $61 \%$ of patients was observed early after introducing dasatinib (17). It is a similar phenomenon observed also after therapy with BTK and PI3K inhibitors, suggesting their shared mechanism of action. Kater et al (17) speculated that lymphocytosis is a result of dasatinib influence on BCR signaling and chemokine-controlled integrin-mediated retention and homing of malignant B cells in lymph node, what explains nodal responses of CLL patients after treatment. The preclinical efficacy of dasatinib is due to inhibition of LYN and SFK kinases in CLL cells, overcoming pro-survival signals from BCR and leading to apoptosis $(8,20,31,32)$. Moreover, several SFK kinases contribute to the regulation of $\mathrm{NK}$ and NK/T cells. 
A
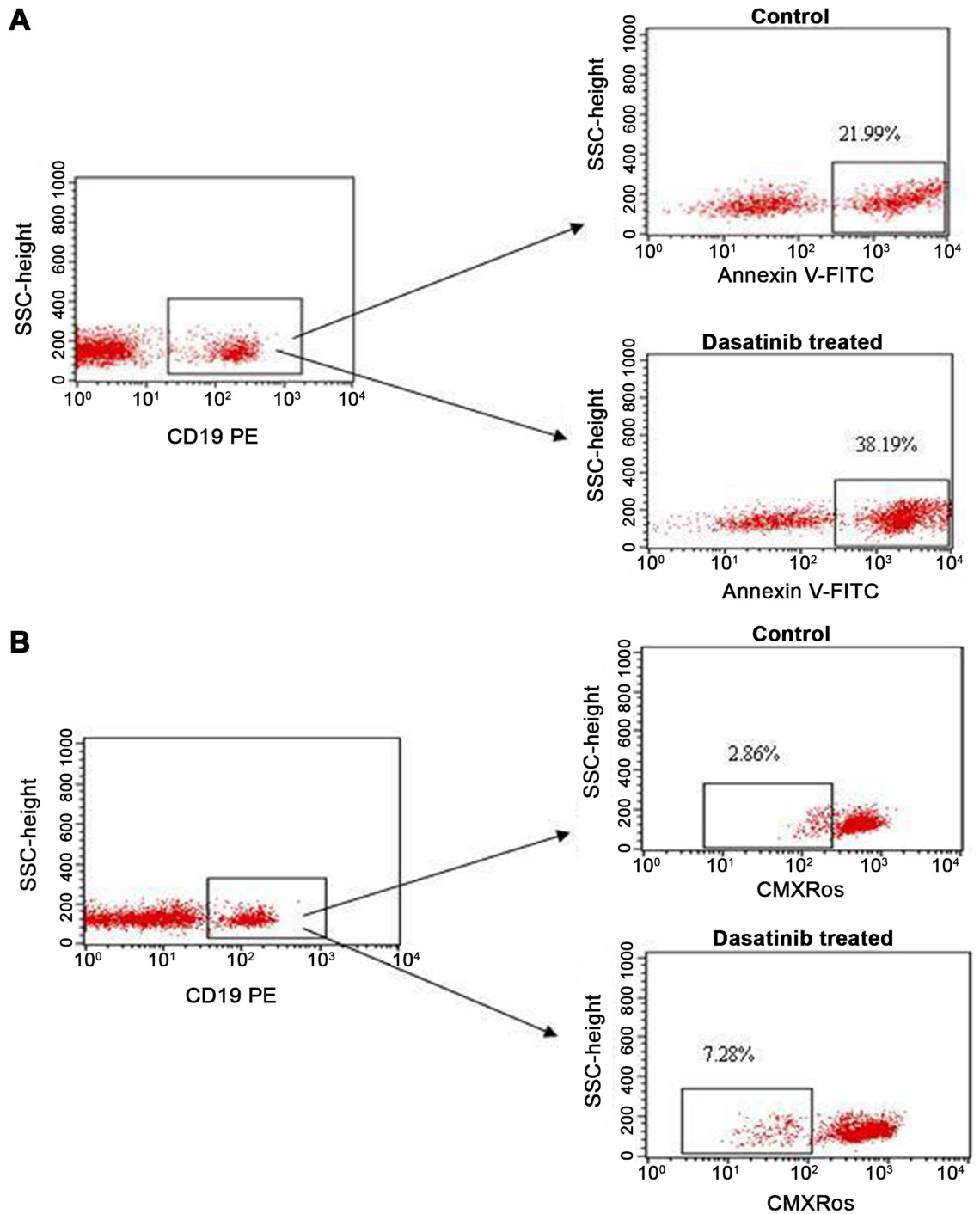

Figure 3. In vitro percentages of apoptotic cells were evaluated by (A) Annexin V and (B) CMXRos in negative control and after dasatinib treatment. A sunsequent analysis on the $\mathrm{CD} 19^{+}$cell population was performed. CMXRos, chloromethyl-X-rosamine; SSC, side scatter.

In CLL/CML patients responding to dasatinib, cytotoxic T and NK large granular lymphocytes were increased (33-35). Although the apoptotic effect of this inhibitor on CLL cells might be diminished by the presence of stromal cells and blood-derived 'nurse-like cells' $(16,28)$.

CLL patients have more than twice the risk of second malignancies development, regardless of treatment status, when compared to the general population (36). In an analysis of patients treated with fludarabine-based protocols in frontline therapy, the risk of other cancers was 2.38 times higher than expected in the general population. Alkylating agents, like cyclophosphamide, did not show evidence of higher risk of second malignancies. Although cyclophosphamide demonstrates weaker carcinogenicity than other alkylating drugs, high doses of this agent might increase the risk of second cancers in CLL and Non-Hodgkin's lymphoma patients (37).

CML coexisting with CLL is a very rare phenomenon, reported in over 20 cases until date. Usually, as in the patient presented here, CML diagnosis follows CLL. Rarely the opposite situation or simultaneous occurrence of CLL and CML was reported. Until date, there is no consensus on the treatment approach for these patients (38). Typically, if CLL was in remission the treatment targeted against CML was introduced. Here, we reported on the patient initially treated with FC, who at the onset of CML was in remission for CLL. Although the patient was in a good prognostic group as characterized by $I G V H$ mutational status, he was not treated optimally with immunochemotherapy including rituximab, we, therefore, might anticipate relapse within next months. In CLL8 clinical trail of German CLL Study Group, patients treated with FC, as compared to those with FCR, have significantly shorter progression-free survival (PFS) of 32.0 vs. 56.8 months (39).

Tecchio et al (40) reported a case of previously untreated CLL patient with trisomy of chromosome 12, a mutated IGVH gene and negative expression of ZAP-70 who developed CML nine years after CLL diagnosis. This patient received $400 \mathrm{mg} /$ day of imatinib. After 2 months, imatinib was discontinued due to skin toxicity and treatment with dasatinib at 100 mg daily was started. Both diseases were monitored after 3 months of therapy, showing a complete hematologic remission and deep molecular response for $B C R-A B L 1$. Similar data concerning a case of CLL patient with a del13q with coexisting CML was published by Nagao et al (41). After 3 months of treatment with dasatinib at a dose of $80 \mathrm{mg} /$ day as the 
first-line therapy, the patient demonstrated a major molecular response for CML and a partial response for CLL characterized by the significant reduction of lymphocyte count (41). Similarly, favorable outcome was observed in our patient with concomitant CML and CLL after 12 months of dasatinib treatment, as the patient exhibited major molecular response. We also observed a reduction of circulating CLL cells pointing to in vivo anti-CLL activity of dasatinib. In 2010, Serpa et al (33) documented a case of CML patient who developed CLL with del11q and positive expression of CD38 in 4th month of imatinib treatment. Based on the literature data suggesting the effectiveness of dasatinib on CLL, the authors changed imatinib to dasatinib at a dose of $100 \mathrm{mg} /$ day. After 6 months, the patient obtained a partial response, with a reduction of lymphocytosis, the disappearance of enlarged lymph nodes, and the maintenance of major molecular responses. Moreover, Pitini et al (29) published case of CLL responsive to treatment with dasatinib at a dose of $140 \mathrm{mg}$ daily in a patient coexisting with relapsed gastrointestinal stromal tumor (GIST).

Results of our study, extended clinical observation with functional analyses of dasatinib induced apoptosis.

In conclusion, dasatinib proved to induce durable antileukemic effects against CML and CLL cells. Its activity could be found in some sensitive CLL cases. Results of this study prove that dasatinib might induce apoptosis in vivo, ex vivo and in vitro in CLL and should represent a preferential therapeutic option for CML/CLL cases.

\section{Acknowledgements}

Not applicable.

\section{Funding}

This work was supported by the Polish National Science Center (grant nos. 2018/31/B/NZ6/03361 and N402 676540) and the Medical University of Lublin (grant no. DS 462).

\section{Availability of data and materials}

All data generated or analyzed during this study are included in this published article.

\section{Authors' contributions}

KG conceived the current study, discussed results and wrote the manuscript. AK and MKa performed the experiments, analyzed and interpreted the data, and wrote the manuscript. ABJ, KK and MM performed the experiments. MKo, WT and MH recruited patients and provided clinical data. TS designed the research and discussed results. $\mathrm{KG}, \mathrm{AK}$ and MKa confirm the authenticity of all the raw data. All authors read and approved the final manuscript.

\section{Ethics approval and consent to participate}

The current study was approved by the Ethics Committee of the Medical University of Lublin (Lublin, Poland; approval nos. KE-0254/269/2019 and KE-0254/305/2019). All patients enrolled in the present study provided their signed informed consent. All forms together with medical history are enclosed in the Experimental Hematooncology Department's database.

\section{Patient consent for publication}

Not applicable.

\section{Competing interests}

The authors declare no competing interests.

\section{References}

1. Ghia P,Ferreri AM and Caligaris-Cappio F: Chronic lymphocytic leukemia. Crit Rev Oncol Hematol 64: 234-246, 2007.

2. Giannopoulos K: Biologia i rokowanie w przewlekłej białaczce limfocytowej. Acta Haematol Pol 41: 433-440, 2010.

3. Zenz T, Mertens D, Döhner H and Stilgenbauer S: Importance of genetics in chronic lymphocytic leukemia. Blood Rev 25: 131-137, 2011.

4. Kuckertz M, Patz M, Veldurthy A, Gehrke I, Claasen J, Frenzel LP, Wendtner CM, Hallek M and Krause G: Comparison of the effects of two kinase inhibitors, sorafenib and dasatinib, on chronic lymphocytic leukemia cells. Onkologie 35: 420-426, 2012.

5. Stevenson FK and Caligaris-Cappio F: Chronic lymphocytic leukemia: Revelations from the B-cell receptor. Blood 103: 4389-4395, 2004.

6. Amrein PC: The potential for dasatinib in treating chronic lymphocytic leukemia, acute myeloid leukemia, and myeloproliferative neoplasms. Leuk Lymphoma 52: 754-763, 2011.

7. Winiarska M, Bojarczuk K, Pyrzynska B, Bil J, Siernicka M, Dwojak M, Bobrowicz M, Miazek N,Zapala P,Zagozdzon A, et al: Inhibitors of SRC kinases impair antitumor activity of anti-CD20 monoclonal antibodies. MAbs 6: 1300-1313, 2014.

8. McCaig AM, Cosimo E, Leach MT and Michie AM: Dasatinib inhibits B cell receptor signalling in chronic lymphocytic leukaemia but novel combination approaches are required to overcome additional pro-survival microenvironmental signals. Br J Haematol 153: 199-211, 2011.

9. Amrein L, Hernandez TA, Ferrario C, Johnston J, Gibson SB, Panasci L and Aloyz R: Dasatinib sensitizes primary chronic lymphocytic leukaemia lymphocytes to chlorambucil and fludarabine in vitro. Br J Haematol 143: 698-706, 2008.

10. Contri A, Brunati AM, Trentin L, Cabrelle A, Miorin M, Cesaro L, Pinna LA, Zambello R, Semenzato G and Donella-Deana A: Chronic lymphocytic leukemia B cells contain anomalous Lyn tyrosine kinase, a putative contribution to defective apoptosis. J Clin Invest 115: 369-378, 2005.

11. Gobessi S,Laurenti L,Longo PG,Sica S,Leone G and Efremov DG: ZAP-70 enhances B-cell-receptor signaling despite absent or inefficient tyrosine kinase activation in chronic lymphocytic leukemia and lymphoma B cells. Blood 109: 2032-2039, 2007.

12. Lin K, Glenn MA, Harris RJ, Duckworth AD, Dennett S, Cawley JC, Zuzel M and Slupsky JR: c-Abl expression in chronic lymphocytic leukemia cells: Clinical and therapeutic implications. Cancer Res 66: 7801-7809, 2006.

13. Hochhaus A, Kantarjian HM, Baccarani M, Lipton JH, Apperley JF, Druker BJ, Facon T, Goldberg SL, Cervantes F, Niederwieser D, et al: Dasatinib induces notable hematologic and cytogenetic responses in chronic-phase chronic myeloid leukemia after failure of imatinib therapy. Blood 109: 2303-2309, 2007.

14. McCaig AM, Cosimo E, Leach MT and Michie AM: Dasatinib inhibits CXCR4 signaling in chronic lymphocytic leukaemia cells and impairs migration towards CXCL12. PLoS One 7: e48929, 2012.

15. Amrein L, Soulières D, Johnston JB and Aloyz R: p53 and autophagy contribute to dasatinib resistance in primary CLL lymphocytes. Leuk Res 35: 99-102, 2011.

16. Hallaert DYH, Jaspers A, van Noesel CJ, van Oers MH, Kater AP and Eldering E: c-Abl kinase inhibitors overcome CD40-mediated drug resistance in CLL: Implications for therapeutic targeting of chemoresistant niches. Blood 112: 5141-5149, 2008. 
17. Kater AP, Spiering M, Liu RD, Doreen Te Raa G, Slinger E, Tonino SH, Beckers MM, Daenen S, Doorduijn JK, Lankheet NA, et al: Dasatinib in combination with fludarabine in patients with refractory chronic lymphocytic leukemia: A multicenter phase 2 study. Leuk Res 38: 34-41, 2014.

18. ten Hacken E, Scielzo C, Bertilaccio MTS, Scarfò L, Apollonio B, Barbaglio F, Stamatopoulos K, Ponzoni M, Ghia P and Caligaris-Cappio F: Targeting the LYN/HS1 signaling axis in chronic lymphocytic leukemia. Blood 121: 2264-2273, 2013.

19. Giannopoulos K, Dmoszynska A, Kowal M, Wasik-Szczepanek E, Bojarska-Junak A, Rolinski J, Döhner H, Stilgenbauer S and Bullinger L: Thalidomide exerts distinct molecular antileukemic effects and combined thalidomide/fludarabine therapy is clinically effective in high-risk chronic lymphocytic leukemia. Leukemia 23: 1771-1778, 2009.

20. Lombardo LJ, Lee FY, Chen P, Norris D, Barrish JC, Behnia K, Castaneda S, Cornelius LAM, Das J, Doweyko AM, et al: Discovery of N-(2-chloro-6-methyl-phenyl)-2-(6-(4(2-hydroxyethyl)-piperazin-1-yl)-2-methylpyrimidin-4-ylamino) thiazole-5-carboxamide (BMS-354825), a dual Src/Abl kinase inhibitor with potent antitumor activity in preclinical assays. J Med Chem 47: 6658-6661, 2004.

21. Martinez Marignac VL, Smith S, Toban N, Bazile M and Aloyz R: Resistance to Dasatinib in primary chronic lymphocytic leukemia lymphocytes involves AMPK-mediated energetic re-programming. Oncotarget 4: 2550-2566, 2013.

22. Amrein PC, Attar EC, Takvorian T, Hochberg EP, Ballen KK Leahy KM, Fisher DC, Lacasce AS, Jacobsen ED, Armand P, et al: Phase II study of dasatinib in relapsed or refractory chronic lymphocytic leukemia. Clin Cancer Res 17: 2977-2986, 2011.

23. Hochhaus A, Baccarani M, Silver RT, Schiffer C, Apperley JF, Cervantes F, Clark RE, Cortes JE, Deininger MW, Guilhot F, et al: European LeukemiaNet 2020 recommendations for treating chronic myeloid leukemia. Leukemia 34: 966-984, 2020.

24. Putowski M, Podgórniak M, Piróg M, Knap J, Zaleska J, Purkot J, Zawiślak J, Zakrzewska E, Karczmarczyk A, Własiuk P, et al: Prognostic impact of NOTCH1, MYD88, and SF3B1 mutations in Polish patients with chronic lymphocytic leukemia. Pol Arch Intern Med 127: 238-244, 2017.

25. Bojarska-Junak A, Giannopoulos K, Kowal M, Dmoszyńska A and Roliński J: Comparison of methods for determining zeta-chain associated protein-70 (ZAP-70) expression in patients with B-cel chronic lymphocytic leukemia (B-CLL). Cytometry B Clin Cytom 70: 293-301, 2006.

26. Burger JA and O'Brien S: Evolution of CLL treatment-from chemoimmunotherapy to targeted and individualized therapy. Nat Rev Clin Oncol 15: 510-527, 2018.

27. Dühren-von Minden M, Übelhart R, Schneider D, Wossning T, Bach MP, Buchner M, Hofmann D, Surova E, Follo M, Köhler F, et al: Chronic lymphocytic leukaemia is driven by antigen-independent cell-autonomous signalling. Nature 489: 309-312, 2012

28. Veldurthy A, Patz M, Hagist S, Pallasch CP, Wendtner CM, Hallek M and Krause G: The kinase inhibitor dasatinib induces apoptosis in chronic lymphocytic leukemia cells in vitro with preference for a subgroup of patients with unmutated $\operatorname{IgVH}$ genes. Blood 112: 1443-1452, 2008.

29. Pitini V, Arrigo C and Altavilla G: Dasatinib induces a response in chronic lymphocytic leukemia. Blood 113: 498, 2009.
30. Garg RJ, Wierda W, Fayad L, Estrov Z, Bickel SM and O'Brien S: Phase II study of dasatinib in patients with relapsed CLL. Blood 112: 4197a, 2008.

31. Song Z, Lu P, Furman RR, Leonard JP, Martin P, Tyrell L, Lee FY, Knowles DM, Coleman M and Wang YL: Activities of SYK and PLCgamma2 predict apoptotic response of CLL cells to SRC tyrosine kinase inhibitor dasatinib. Clin Cancer Res 16: 587-599, 2010.

32. Hantschel O, Rix U, Schmidt U, Bürckstümmer T, Kneidinger M, Schütze G, Colinge J, Bennett KL, Ellmeier W, Valent P, et al: The Btk tyrosine kinase is a major target of the Bcr-Abl inhibitor dasatinib. Proc Natl Acad Sci USA 104: 13283-13288, 2007.

33. Serpa M, Bendit I, Seguro F, Xavier F, Cavalcante M, Steinbaum D, Nardinelli L, Aldred VL, de Paula HM and Dorlhiac-Llacer PE: Response to dasatinib in a patient with concomitant chronic myeloid leukemia and chronic lymphocytic leukemia. Acta Haematol 124: 105-109, 2010.

34. Kim DH, Kamel-Reid S, Chang H, Sutherland R, Jung CW, Kim HJ, Lee JJ and Lipton JH: Natural killer or natural killer/T cell lineage large granular lymphocytosis associated with dasatinib therapy for Philadelphia chromosome positive leukemia. Haematologica 94: 135-139, 2009.

35. Mustjoki S, Ekblom M, Arstila TP, Dybedal I,Epling-Burnette PK, Guilhot F, Hjorth-Hansen H, Höglund M, Kovanen P, Laurinolli T, et al: Clonal expansion of T/NK-cells during tyrosine kinase inhibitor dasatinib therapy. Leukemia 23: 1398-1405, 2009.

36. Tsimberidou AM, Wen S, McLaughlin P, O'Brien S, Wierda WG, Lerner S, Strom S, Freireich EJ, Medeiros LJ, Kantarjian HM, et al: Other malignancies in chronic lymphocytic leukemia/small lymphocytic lymphoma. J Clin Oncol 27: 904-910, 2009.

37. Benjamini O, Jain P, Trinh L, Qiao W, Strom SS, Lerner S, Wang X, Burger J, Ferrajoli A, Kantarjian H, et al: Second cancers in patients with chronic lymphocytic leukemia who received frontline fludarabine, cyclophosphamide and rituximab therapy: Distribution and clinical outcomes. Leuk Lymphoma 56: 1643-1650, 2015

38. Boddu P, Gibbons J, Burger J, Sivina M, Thakral B, Kanagal-Shamanna R and Ferrajoli A: Co-occurrence of chronic myeloid leukemia with chronic lymphocytic leukemia: A report of two cases. Leuk Lymphoma 60: 1568-1571, 2019.

39. Fischer K, Bahlo J, Fink AM, Goede V, Herling CD, Cramer P, Langerbeins P, von Tresckow J, Engelke A, Maurer C, et al: Long-term remissions after FCR chemoimmunotherapy in previously untreated patients with CLL: Updated results of the CLL8 trial. Blood 127: 208-215, 2016.

40. Tecchio C, Nichele I, Todeschini G, Pizzolo G and Ambrosetti A: Dasatinib-induced response in a rare case of chronic lymphocytic leukaemia associated with chronic myeloid leukaemia. Br J Haematol 146: 222-223, 2009.

41. Nagao T, Takahashi N, Kameoka Y, Noguchi S, Shinohara Y, Ohyagi H, Kume M and Sawada K: Dasatinib-responsive chronic lymphocytic leukemia in a patient treated for coexisting chronic myeloid leukemia. Intern Med 52: 2567-2571, 2013.

This work is licensed under a Creative Commons Attribution-NonCommercial-NoDerivatives 4.0 International (CC BY-NC-ND 4.0) License. 